\title{
Endomiocardiofibrose: resultados do tratamento cirúrgico com conservação das valvas atrioventriculares
}

\author{
Sérgio Almeida de OLIVEIRA*, Luís Alberto DALLAN*, Antônio Carlos PEREIRA-BARRETO*, Charles \\ MADY*, Fúlvio PILEGGI*, Adib D. JATENE*
}

RBCCV 44205-125

\begin{abstract}
Oliveira, S. A.; DALlan, L. A.; PEREIRA-BARRETO, A. C.; MADY, C.; PILEgGI, F.; JATENE, A. D. - Endomiocardiofibrose: resultados do tratamento cirúrgico com conservação das valvas atrioventriculares. Rev. Bras. Cir. Cardiovasc., 6(1): 3-10, 1991.
\end{abstract}

RESUMO: Entre abril de 1988 e janeiro de 1991, foram operados 25 pacientes consecutivos, para correção de endomiocardiofibrose, com técnica conservadora das valvas atrioventriculares. Dezenove pacientes eram do sexo feminino e seis do masculino. As idades variaram de 11 a 59 anos, com média de 40,6 anos. Dezesete pacientes apresentavam acometimento biventricular, seis com comprometimento do ventrículo esquerdo e apenas dois pacientes com lesão isolada do ventrículo direito. Todos estavam em grupos avançados de insuficiência cardiaca, sendo $19 \mathrm{em}$ grupo IV da NYHA e seis no grupo III. No pré-operatório, 24 apresentavam insuficiência da valva tricúspide e 22 apresentavam insuficiência da valva mitral. Todos os pacientes foram operados com o auxílio de circulação extracorpórea e hipotermia sistêmica de $28^{\circ} \mathrm{C}$ e pinçamento aórtico intermitente, para melhor exposição das cavidades ventriculares. A fibrose ventricular direita foi sempre ressecada através da valva tricúspide, enquanto que a fibrose do ventrículo esquerdo foi removida através de pequena ventriculotomia esquerda apical. Em todos os pacientes com insuficiência tricúspide, foi feita plástica anular do tipo DeVega. Dos pacientes com insuficiência mitral, sete tiveram correção espontânea após a ressecção da fibrose, e os outros 15 receberam anuloplastia. Houve apenas um (4\%) óbito no pós-operatório imediato e nenhum óbito tardio. A recuperação funcional foi boa, estando atualmente 16 pacientes em grupo funcional I e $8 \mathrm{em}$ grupo II.

DESCRITORES: endomiocardiofibrose, cirurgia; valvas cardiacas, atrioventriculares, cirurgia; miocardiopatias, cirurgia.

\section{INTRODUÇĀO}

A cirurgia para correção da endomiocardiofibrose (EMF) iniciou-se no Instituto do Coração em 1987, sendo, desde então, operados 77 pacientes. Parte desta casuistica já foi relatada em trabalhos anteriores " ". Até 1988, utilizamos a técnica descrita, em 1971, por DUBOST et alii ${ }^{6}$. Em abril de 1988, iniciamos nova técnica visando preservar em valvas atrioventriculares ${ }^{16,17}$, sem prejuizo da ressecção da fibrose ventricular. Não há referência, na literatura médica, de recidiva da endomiocardiofibrose após a ressecção cirúrgica e as reoperaçōes realizadas foram indicadas para a correção de disfunção das próteses, ou biopróteses utilizadas para a substituiçâo das valvas atrioventiculares. Por outro lado, o acometimento valvar geralmente se restringe a fibrose que envolve a base dos músculos papilares e só muito raramente acomete, as cúspides valvares e ainda assim quando

Trabalho realizado no Instituto do Coraçāo do Hospital das Clinicas da Faculdade de Medicina da Universidade de São Paulo. São Paulo, SP. Brasil.

Apresentado ao 18: Congresso Nacional de Cirurgia Cardiaca. Rio de Janeiro, RJ, 5 e 6 de abril, 1991.

Laureado com o "Prêmio Nacional de Cirurgia Cardiaca - 1991".

- Do Instituto do Coração do Hospital das Clinicas da Faculdade de Medicina da Universidade de Săo Paulo.

Endereço para separatas: Sérgio Almeida de Oliveira. Av. Dr. Enéas de Carvalho Aguiar, 44. Divisảo Cirúrgica. 05403 Sảo Paulo, SP, Brasil. 
OliveIRA, S. A.; DALlan, L. A.; PEREIRA-BARRETO, A. C.; MADY, C.; PILEGGI, F.; JATENE, A. D. - Endomiocardiofibrose: resultados do tratamento cirúrgico com conservação das valvas atrioventriculares. Rev. Bras. Cir. Cardiovasc., 6(1): 3-10, 1991.

TABELA 1

DISTRIBUICATO DO NÜMERO DE PACIENTES OPERADOS COM A TÉCNICA CLÁSSICA E A TÉCNICA PROPOSTA PELOS AUTORES.

\begin{tabular}{lcl}
\hline Periodo & $\begin{array}{c}\text { N. pacientes } \\
\text { operados }\end{array}$ & \multicolumn{1}{c}{ Técnica } \\
\hline $1978-1987$ & 52 & Clássica (Dubost) \\
$1988-1991$ & 25 & Com preservação valvar \\
\hline
\end{tabular}

$\mathrm{n}=$ número de pacientes.

isto ocorre, a cúspide acometida é a mural ou posterior da valva mitral, ou a septal da valva tricúspide ${ }^{7,}{ }^{19}$, tornando mais fácil a realizaçāo de uma cirurgia conservadora.

Neste trabalho, relatamos a experiência adquirida com a utilização desta técnica em 25 pacientes consecutivos (Tabela 1).

\section{CASUÍSTICA E MÉTODOS}

Entre abril de 1988 e janeiro de 1991, foram operados 25 pacientes com o diagnóstico de endomiocardiofibrose. A idade dos pacientes variou de 11 a 59 anos, com média de 40,6 anos. Dezenove pacientes eram do sexo feminino e seis do sexo masculino. A EMF acometia ambos os ventrículos em 17 pacientes. Estava localizada apenas no ventrículo esquerdo em seis pacientes e somente no ventrículo direito em dois. Todos os pacientes apresentavam insuficiência cardiaca, estando, no momento da operaçāo, em grupo funcional IV da NYHA 19 pacientes e no grupo III seis pacientes. Insuficiência da valva tricúspide estava presente em 24 pacientes

TABELA 2

RELACÃO DAS CARACTERISTICAS GERAIS DOS PACIENTES COM EMF

ENDOMIOCARDIOFIBROSE
TRATAMENTO CIRÜRGICO
CARACTERISTICAS GERAIS DOS PACIENTES
$\mathrm{n}=25$

Idade: 11 a 59 anos ( $m=40,6$ anos)

Sexo: Feminino 19

Masculino 06

Localização da EMF - Biventricular 17

V. esquerdo 06

V. direito 02

Classe Funcional - CF IV 19

(Pré-Operatório) CF III 06

(NYHA)

$\mathrm{n}=$ número de pacientes; $\mathrm{m}=$ meses.

$\mathrm{CF}=$ classe funcional; NYHA $=$ New York Heart Association. e insuficiência da valva mitral em 22. Como doenças associadas, observamos doença obstrutiva coronária em um paciente, doença de Behcet em um e esquistossomose mansônica em um. Ascite e derrames pleurais foram observados em vários pacientes, exigindo drenagens repetidas (Tabela 2 ).

O diagnóstico da EMF foi suspeitado com base clínica pelos sinais e sintomas e complementado pelos estudos ecocardiográficos e cineangiocardiográficos realizados em todos os pacientes. Nos últimos dois pacientes, foram realizados estudos com ressonância magnética nuclear. Cinecoronariografia foi também realizada em todos os pacientes. Todos permaneceram internados por períodos variáveis no pré-operatório, buscando compensá-los da insuficiência cardíaca. Acentuada desnutrição foi observada em muitos deles com insuficiência cardiaca de longa duração. Nestes casos, foi realizada, no pré-operatório, alimentação enteral com auxilio de sonda de Duboff.

A operação foi realizada com acesso ao coração por via mediana transesternal. Em todos os casos, foi utilizada circulação extracorpórea, usando-se oxigenadores de bolhas ou membranas, hemodiluição com solução de Ringer-lactato e hipotermia sistêmica de $28^{\circ} \mathrm{C}$ (temperatura esofágica). Pinçamento aórtico intermitente por períodos de dez minutos, intercalados com reperfusāo coronária de três a quatro minutos, foi utilizado em todos os pacientes, durante a remoção da fibrose ventricular e da correção da disfunção valvar. A técnica para a correção da EMF foi aquela já descrita anteriormente. As valvas atrioventriculares foram analisadas por via atrial, antes da ressecção da fibrose ventricular. Trombose atrial direita foi encontrada em quatro pacientes, sendo os trombos removidos logo após a atriotomia. A trombose estava localizada na aurícula direita, mas, em três pacientes, o trombo era muito volumoso, ocupando grande parte do átrio. Trombose atrial esquerda foi encontrada em apenas um paciente, de pequenas dimensões localizada na aurícula esquerda. A valva tricúspide estava insuficiente em 24 pacientes. Em todos havia grande dilataçāo do anel da valva tricúspide. Nos casos com EMF do ventrículo direito havia, porém, envolvimento dos músculos papilares pela fibrose, especialmente nas bases. A insuficiência da valva tricúspide foi, em todos os pacientes, tratada com a anuloplastia de DeVega ${ }^{5}$.

Insuficiência da valva mitral foi observada em 22 pacientes. Após a ressecção da fibrose do ventrículo esquerdo, houve desaparecimento da insuficiência mitral em sete pacientes. Nos outros 15 pacientes com regurgitação residual, embora reduzida após a remoçāo da fibrose, foram realizadas plásticas para a correção da insuficiência mitral. No primeiro paciente operado com esta técnica para remoção da EMF do ventrículo esquerdo, a redução do anel mitral foi realizada com a colocação de um anel de Carpentier (CARPENTIER et alii ${ }^{2}$ ). Em 
OlIVEIRA, S. A.; DALLAN, L. A.; PEREIRA-BARRETO, A. C.; MADY, C.; PILEGGI, F.; JATENE, A. D. - Endomiocardiofibrose: resultados do tratamento cirúrgico com conservação das valvas atrioventriculares. Rev. Bras. Cir. Cardiovasc., 6(1): 3-10, 1991.

nove casos que se seguiram, foi realizada anuloplastia tipo Kay (KAY et alii $^{9}$ ) em ambas as comissuras. Um destes pacientes apresentava, na cúspide posterior, próxima à comissura látero-posterior, rotura de três cordas tendíneas, sendo realizada ressecção quadrangular, com reconstrução da cúspide e redução do anel. O exame histopatológico do segmento removido da valva mitral revelou degeneração mucóide do tecido. Nos últimos seis pacientes, realizamos a redução do anel com fixação de um pequeno retalho de pericárdio heterólogo, recortado em forma de $\mathrm{C}$ e aplicado sobre o hemi-anel posterior.

A ressecçāo da EMF de ambos os ventrículos obedeceu à sistematização técnica já descrita por nós em outros trabalhos ${ }^{16,17}$. Entretanto, nos últimos nove pacientes com EMF do ventrículo esquerdo, a ventriculotomia esquerda foi fechada com dupla linha de sutura com fio de Polipropileno, sem reforço com tiras de feltro de Dacron ancorando os pontos externamente.

Cateter de Swan-Ganz introduzido por punção de uma das veias subclávia ou jugular interna foi sempre utilizado para controle hemodinâmico no pós-operatório imediato. Eletrodos de marcapasso externo foram também colocados em todos os pacientes.

No paciente com lesão obstrutiva coronária, foi realizada anastomose da artéria torácica interna esquerda com a artéria interventricular anterior. O paciente com doença de Behcet apresentava um grande trombo aderente à valva tricúspide, além de trombos no tronco pulmonar e artéria pulmonar esquerda, tendo sido removidos.

A comparação das médias das variáveis pré e pós-operatórias foi realizada através do teste $T$ de Student pareado.

\section{RESULTADOS}

\section{Mortalidade e seguimento pós-operatório}

Houve um (4\%) óbito no pós-operatório imediato. Era uma paciente com EMF biventricular com longa história de insuficiência cardiaca congestiva, ascite de grande magnitude e caquexia. A paciente evoluiu após a operação com baixo débito cardíaco, refazendo rapidamente a ascite e derrames pleurais, falecendo no 9 : dia de pós-operatório. A necropsia mostrou completa remoção da fibrose endocárdica de ambos os ventrículos, com valva mitral competente e discreta insuficiência tricúspide. Não houve óbitos tardios, num seguimento médio de 14,7 meses, variando de dois a 32 meses.

\section{Evolução clínica}

Todos os pacientes apresentaram melhora clínica.
TABELA 3

EVOLUÇĀO DA CLASSE FUNCIONAL DOS PACIENTES OPERADOS PARA CORREÇÃO DA EMF

\begin{tabular}{cc}
\hline $\begin{array}{c}\text { Classe } \\
\text { funcional } \\
\text { (NYHA) }\end{array}$ & Número de pacientes \\
\cline { 2 - 2 } II & Pré-Op \\
\hline III & -
\end{tabular}

* 1 óbito imediato

Seguimento pos-op = 14,7 m (2 a 32 meses)

NYHA = New York Heart Association; pré-op = pré-operatório; pós-op = pos-operatório; $m=$ meses.

Dos seis pacientes que estavam em grupo funcional III no pré-operatório, dois passaram para o grupo II e quatro passaram para o grupo I. Dos 19 pacientes que estavam no grupo IV, um faleceu no pós-operatório imediato, seis passaram para o grupo II e 12 passaram para o grupo I (Tabela 3) da classificaçāo da NYHA. A administração de digital, diuréticos, ou de ambos, foi realizada em todos os pacientes após a operação, mas podendo ser suspensa na maioria deles após alguns meses de pós-operatório. A melhora clínica foi mais rápida nos casos com predomínio da fibrose no ventrículo esquerdo. Não houve bloqueio atrioventricular total em nenhum paciente. Um paciente desenvolveu síndrome pós pericardiotomia com tamponamento cardíaco, sendo necessária drenagem cirúrgica. Esta mesma paciente apresentou, no pós-operatório imediato, icterícia de etiologia não identificada, mas que regrediu após quatro semanas, evoluindo muito bem desde então.

\section{Resultado hemodinâmico}

Estudos hemodinâmicos e cineangiográficos foram realizados em 14 pacientes, antes da alta hospitalar. Estes estudos (Figura 1) mostraram significativa reduçāo das pressões médias de ambos os átrios e das pressōes diastólicas finais de ambos os ventrículos, quando comparadas com as pressōes determinadas nos estudos pré-operatórios. Também foi observada significativa redução nas pressōes sistólicas e diastólicas do tronco pulmonar. Estas pressōes estavam elevadas na maioria dos pacientes e diminuíram após a operação (Figura 2). A Figura 3 mostra a angiografia de um caso de EMF do ventrículo direito, antes e após a remoção da fibrose ventricular e após a correção da insuficiência tricúspide. A Figura 4 mostra a cineangiografia de um caso de EMF do ventrículo esquerdo, antes e após a remoção da fibrose e a correção da insuficiência mitral. $O$ aspecto angiográfico do ventrículo esquerdo torna-se quase normal, enquanto o ventrículo direito, embora melhore a contrati- 
OLIVEIRA, S. A.; DALLAN, L. A.; PEREIRA-BARRETO, A. C.; MADY, C.; PILEGGI, F.; JATENE, A. D. - Endomiocardiofibrose: resultados do tratamento cirúrgico com conservaçāo das valvas atrioventriculares. Rev. Bras. Cir. Cardiovasc., 6(1): 3-10, 1991.

lidade, permanece ainda alterado, principalmente pela ausência da zona trabecular. Estudos ecocardiográficos têm sido realizados periodicamente a cada seis meses, sendo muito útil para o acompanhamento pós-operatório. Os dois últimos pacientes desta séria foram submetidos a estudo com ressonância magnética nuclear. Este estudo tem grande sensibilidade para mostrar as áreas de fibrose miocárdica.
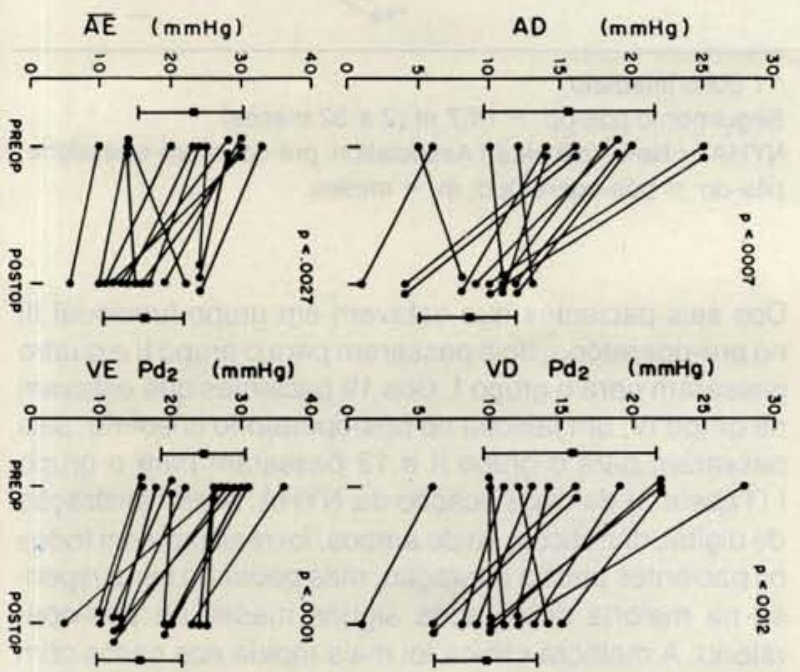

Fig. 1 - Representação gráfica da evolução pré- e pós-operatória das pressōes médias dos átrios direito e esquerdo e das pressōes sistólicas finais dos ventriculos direito e esquerdo. $\mathrm{AE}=$ átrio esquerdo; $A D=$ átrio direito; $V E=$ ventriculo esquerdo; $V D$ $=$ ventrículo direito; pré-op $=$ pré-operatório; $p o s-o p=p o ́ s-0$ peratório.
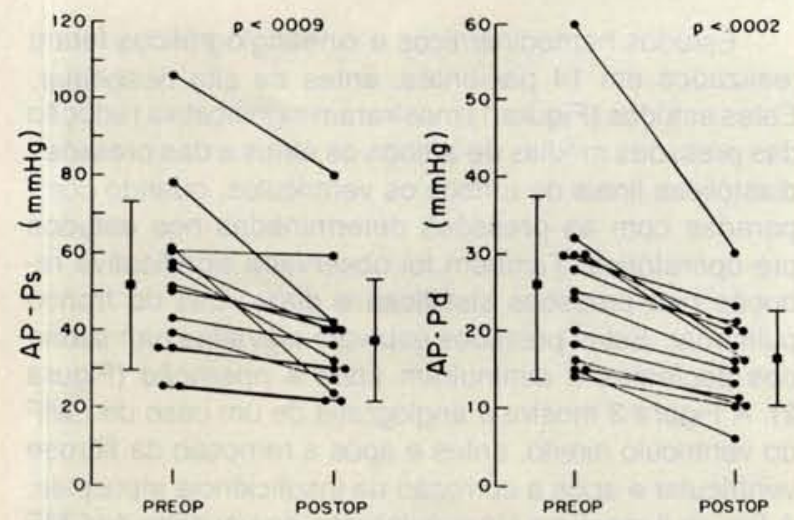

Fig. 2-Representação gráfica da evolução das pressōes sistólica e diastólica da artéria pulmonar antes e após a operação. Ap-Ps = artéria pulmonar, pressảo sistólica; $\mathrm{AP}-\mathrm{Pd}=$ artéria pulmonar na pressão diastólica; pré-op = pré-operatório; pósop $=$ pós-operatório.

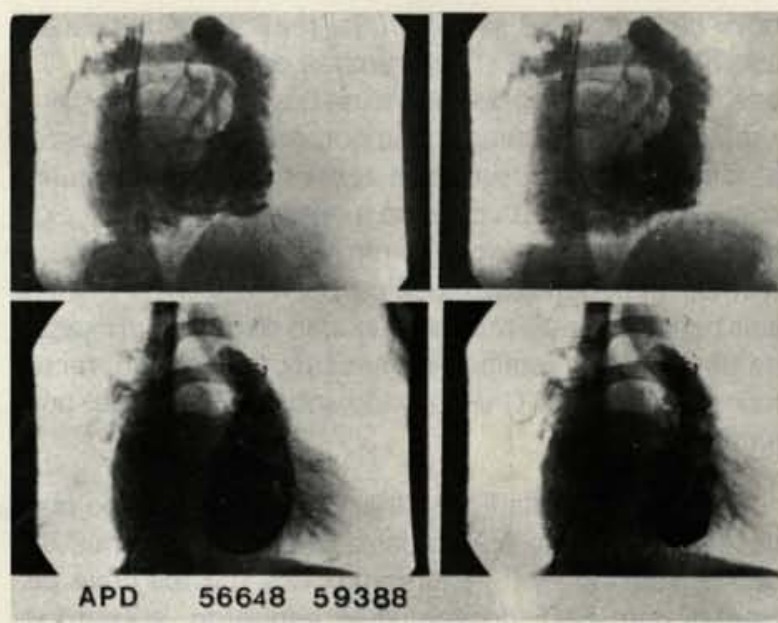

Fig. 3-Cineangiografia pré e pós-operatória de um paciente com EMF do ventrículo direito.

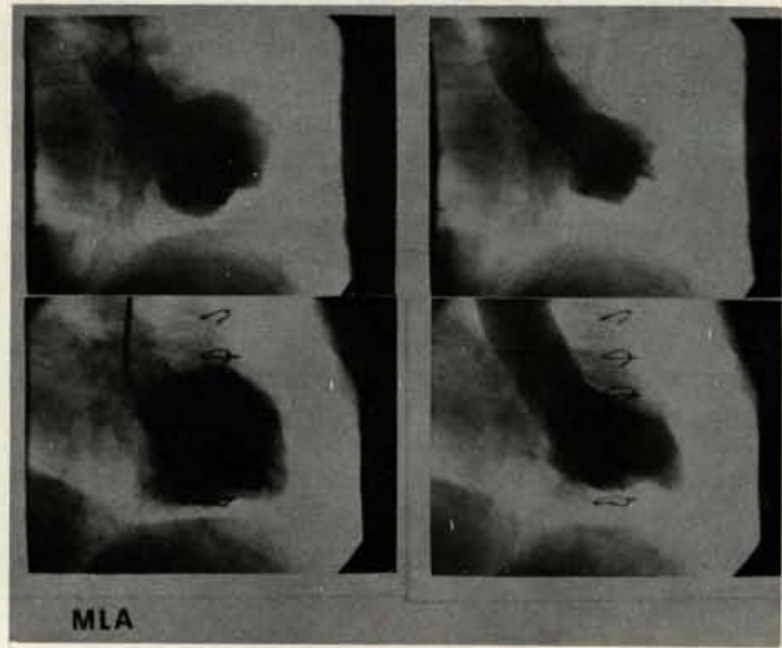

Fig. 4-Cineangiografia pré e pós-operatória de um paciente com EMF do ventriculo esquerdo.

\section{COMENTÁRIOS}

$\mathrm{O}$ acometimento das valvas atrioventriculares na EMF restringe-se, na quase totalidade dos casos, a fibrose que envolve os músculos papilares, especialmente em suas porções basais. Lesões das cúspides são raras e, quando ocorrem, localizam-se nas cúspides septal ou posterior da valva tricúspide, ou na cúspide posterior da valva mitral ${ }^{4,5}$. Estas peculiaridades da EMF propiciam o tratamento conservador destas valvas durante a correção cirúrgica. Entretanto, na literatura, observamos que a remoção e substituição valvar têm sido realizadas na maioria dos casos relatados ${ }^{7},{ }^{19}$, ficando 0 tratamento conservador das valvas restrito às formas mais localizadas da doença. Por outro lado, sabemos 
Oliveira, S. A.; DALlAN, L. A.; PEREIRA-BARRetO, A. C.; MADY, C.; PILEGGI, F.; JATENE, A. D. - Endomiocardiofibrose: resultados do tratamento cirúrgico com conservação das valvas atrioventriculares. Rev. Bras. Cir. Cardiovasc., 6(1): 3-10, 1991.

que os substitutos valvares ainda apresentam muitos problemas $^{18}$, o que torna desejável, sempre que possivel, preservar as valvas cardiacas.

A técnica que descrevemos para a correção da EMF foi empregada nesta série consecutiva de 25 pacientes, sendo possível, em todos, preservar as valvas mitral e tricúspide. Acreditamos que a disfunção valvar é, primeiramente, devida à distorção dos músculos papilares pela fibrose endocárdica e, secundariamente, devida à dilatação do anel valvar.

A etiologia da EMF ainda não é bem conhecida, mas a recidiva da fibrose endocárdica não tem sido descrita após o tratamento cirúrgico $4,13,14$. Assim sendo, as reoperaçōes que foram realizadas em nossa série anterior e também por outros autores ${ }^{11,13,14}$ foram motivadas pela disfunção dos substitutos valvares utilizados na primeira operaçāo, e não por recidiva da EMF.

A via venticular para a remoção da fibrose do ventrículo esquerdo foi raramente utilizada, mas quase sempre associada a remoção e substituição valvar ${ }^{12,13},{ }^{15}$. MORAES et alii ${ }^{15}$ utilizaram a via ventricular esquerda em casos com fibrose localizada e com regurgitação mitral ausente ou de leve intensidade. METRAS et alii ${ }^{13}$ não recomendam a via ventricular esquerda. Acreditam ser desnecessária, além de acarretar dano adicional ao miocárdio. Entretanto, estes autores utilizaram a via ventricular associada à remoção valvar mitral.

Nós modificamos a técnica, com a finalidade de permitir a completa remoção da fibrose endocárdica de ambos os ventrículos, assim como preservar as valvas atrioventriculares. Esta técnica tem sido indicada e realizada em todos os casos, independentemente da extensão e da intensidade da fibrose endocárdica, ou da magnitude da insuficiência valvar.

A mortalidade imediata tem sido elevada, na maioria das séries relatadas ${ }^{3}, 11,13,14$. Em recente revisão da literatura, MORAES ${ }^{14}$ coletou 287 relatos de pacientes operados, com mortalidade imediata de $18,8 \%$ e tardia de $11,2 \%$. No Instituto do Coração do HC-FMUSP, a mortalidade imediata $(20,3 \%)$ e tardia $(10,1 \%)$ com a técnica clássica foi também muito elevada, embora com redução, nos últimos anos desta série, para 10,3\% (imediata) e $3,4 \%$ (tardia) ${ }^{11}$. Com a nova técnica adotada, houve acentuada reduçāo da mortalidade imediata $(4,0 \%)$, mesmo considerando que foram operados somente pacientes em classes III e IV da NYHA. Além da diminuição do risco operatório, observamos melhor evolução clínica e hemodinâmica pós-operatória.

Alguns autores ${ }^{13,14}$ têm proposto que a indicação cirúrgica seja feita em estágios mais precoces da doença, visando diminuir o risco operatório e melhorar os resultados tardios. O acompanhamento clínico de um grupo de pacientes em nosso Instituto ${ }^{1}$ tem mostrado que a evolução guarda nítida correlação com a classe funcional quando da primeira consulta, sendo boa a evolução dos pacientes com classe I e II, enquanto os pacientes já admitidos em classes III e IV apresentam elevadas taxas de mortalidade. Por esta razão, temos mantido a indicação para o tratamento cirúrgico restrita aos pacientes muito sintomáticos. Mas, com a melhora dos resultados cirúrgicos observados nesta série, aliada à alta probabilidade de ser realizado tratamento conservador das valvas atrioventriculares, é possível que este critério possa ser mudado num futuro próximo.

\section{CONCLUSŌES}

A técnica conservadora ${ }^{16}$ utilizada para a correçāo cirúrgica da endomiocardiofibrose é de fácil realização, é aplicável a todos os pacientes e acarreta baixa morbidade e mortalidade. Permite a preservaçāo das valvas atrioventriculares. Restabelece a função ventricular. Como as reoperaçōes na EMF são motivadas pela disfunção das próteses valvares, esta nova técnica é possivelmente preferível, pois preserva a valva original. Embora os resultados sejam encorajadores, um seguimento pósoperatório mais longo é ainda necessário. 
Oliveira, S. A.; DALlan, L. A.; PeReIRA-BARReto, A. C.; MADY, C.; PILEGGI, F.; JATENE, A. D. - Endomiocardiofibrose: resultados do tratamento cirúrgico com conservaçāo das valvas atrioventriculares. Rev. Bras. Cir. Cardiovasc., 6(1): 3-10, 1991.

RBCCV 44205-125

Oliveira, S. A.; DALlan, L. A.; PEREIRA-BARRETO, A. C.; MADY, C.; PILEGGI, F.; JATENE, A. D. - Endomyocardial fibrosis: results of the surgical treatment with atrioventricular valve preservation. Rev. Bras. Cir. Cardiovasc., 6(1): 3-10, 1991.

ABSTRACT: Between April 1988 and January 1991 we operated on 25 consecutive patients with endomyocardial fibrosis (EMF) with an approach to preserve the atrioventricular valves. Nineteen patients were females and six males. Their ages ranged from 11 to 5 years, with an average of 40.6 years. Seventeen patients had biventricular involvement, six had involvement of the left ventricle and only two patients had an isolated lesion of the right ventricle. All of them were in advanced stage of heart failure, 19 of which were in class IV of the NYHA, and six were in class III. Prior to the operation, tricuspid insufficiency was present in 24 patients and mitral insufficiency was present in 22 patients. All the patients were operated on with the support of extracorporeal circulation and systemic hipothermia of $28^{\circ} \mathrm{C}$. Intermitent aortic cross-clamping was used so as to permit better exposition of the ventricular cavities. The fibrosis of the right ventricle was always removed through the tricuspid valve, while the fibrosis of the left ventricle was removed through a small apical left ventriculotomy in all the patients. An DeVega's annuloplasty of the tricuspid valve was necessary in all the patients with tricuspid insuffiency. On the other hand, seven patients had spontaneous correction of the ventricle insufficiency after the removal of the fibrosis of the left ventricle, while fifteen needed an annuloplasty. There was one (4.0\%) in-hospital death and no late deaths. All patients experienced clinical improvement and are in NYHA functional class I or II.

DESCRIPTORS: endomyocardial fibrosis, surgery; heart valves, atrioventricular; cardiomyopathies, surgery.

\section{REFERÊNCIAS BIBLIOGRÁFICAS}

1 BARRETTO, A. C. P.; LUZ, P. L.; MADY, C.; VIANNA, C. B.; JATENE, A. D.; BELLOTTI, G.; PILEGGI, F. - Determinants of survival in patients (pst) with endomyocardial fibrosis (EMF). Circulation, 78 (Supl. 2): 526-530, 1988.

2 CARPENTIER, A.; CHAUWARD, S.; FABIANI, J. N. Reconstructive surgery of mitral valve incompetence. J. Thorac. Cardiovasc. Surg., 79: 338-344, 1980.

3 COSTA, F. D. A.; MORAES, C. R.; RODRIGUES, J. V.; MENDONÇA, J. T.; ANDRADE, J. C.; BUFFOLO, E.; SUCCI, J. E.; CARVALHO, R. G.; FARACO, D. L.; COSTA, I. A. - Eur. J. Cardio-thorac. Surg., 3: 408-413, 1989.

4 DELOCHE, A.; CHAPELON. C.; LEBAIS, V.; DUBOST, C.; CARPENTIER, A. - Long-term result of the surgical treatment of endomyocardial fibrosis. Eur. Heart J., 9 (Supl. 2): 287, 1988. (Resumo).

5 DeVEGA, N. G. - La anuloplastia selectiva, regulable y permanente. Rev. Esp. Cardido, 25: 555-560, 1972.

6 Dubost, C.; MAURiCE, P.; GeRBauX, A.; RULLIERE, R.; BARILLON, A.; BERTRAND, E.; VIAL, F.; PRIGENT, S.; CARPENTIER, A.; SOYER, R. - L'endocardite fibreuse constrictive: traitement chirurgical. Arch. Mal. Coeur, 70: 155-161, 1977.

7 FERRER-BROWN, G. \& TARBIT, M. H. - Heart valve involvement in endomyocardial fibrosis. Br. Heart. J., 34: 1062-1071, 1972.
8 JOHN, S.; MANI, G. K.; MURALIDHARAN, N.; KRISHNASWAMY, S.; CHERIAN, G. - Endomyocardial fibrosis from a surgical standpoint. J. Thorac. Cardiovasc. Surg., 80: 437-440, 1980.

9 KAY, J. H.; ZUBIATE, P.; MENDEZ, M. A. - Mitral valve repair for significant mitral insufficiency. Am. Heart J., 96: 253-268, 1978.

10 LEPLEY Jr., D.; ARIS, A.; KORNS, M. E.; WALKER, J. A.; O'CUNHA, R. M. - Endomyocardial fibrosis: a surgical approach. Ann. Thorac. Surg., 18: 626-633, 1974.

11 MADY, C.; BARRETTO, A. C. P.; OLIVEIRA, S. A.; STOLF, N. A. G.; BELLOTTI, G.; JATENE, A. D.; PILEGGI, F. - Endomiocardiofibrose: evolução de pacientes com tratamento clínico e cirúrgico. Arq. Bras. Cardiol., 55: 241-244, 1990.

12 MENDONÇA, J. T.; CARVALHO, M. R.; COSTA, R. K.; FRANCO FILHO, M. L. B.; COSTA, G. B.; ALMEIDA, M. L. D.; SOUZA, A. O. - Endomiocardiofibrose: tratamento cirúrgico. Arq. Bras. Cardiol., 52: 13-17, 1989.

13 metras, D.; COUlibaly, A. O.; QUATTARA, K. - The surgical treatment of endomyocardial fibrosis: results in 55 patients. Circulation, 72 (Supl. 2): 274-279, 1975.

14 MORAES, C. R. - Early and late results of surgery for endomyocardial fibrosis. In: Eckhardt G. J. \& Olsen, M. R. Restrictive cardiomyopathy and arrhythmias. Tokyo, University of Tokyo Press, 1990. p. 49-57. 
OliveIRA, S. A.; DALLAN, L. A.; PEREIRA-BARRETO, A. C.; MADY, C.; PILEGGI, F.; JATENE, A. D. - Endomiocardiofibrose: resultados do tratamento cirúrgico com conservaçāo das valvas atrioventriculares. Rev. Bras. Cir. Cardiovasc., 6(1): 3-10, 1991.

15 MORAES, C. R.; BUFFOLO, E.; LIMA, R.; VICTOR, E.; LIRA, V.; ESCOBAR, M.; RODRIGUES, J.; SARAIVA, L.; ANDRADE, J. C. - Surgical treatment of endomyocardial fibrosis. J. Thorac. Cardiovasc. Surg., 85: 738-745, 1983

16 OLIVEIRA, S. A.; BARRETTO, A. C. P.; MADY, C.; DALLAN, L. A. O.; LUZ, P. L.; JATENE, A. D.; PILEGGI, F. - Surgical treatment of endomyocardial fibrosis: a new approach. J. Am. Coll. Cardiol., 16:1246-1251, 1990.

17 OLIVEIRA, S. A.; BARRETTO, A. C. P.; PILEGGI, F.; JATENE, A. D. - Endomiocardiofibrose: uma nova abordagem cirúrgica. Arq. Bras. Cardiol., 54: 157, 1990.

18 RAHIMTOOLA, S. H. - Lessons learned about the determinants of the results of valve surgery. Circulation, 78 : 1503-1507, 1988.

19 VIOLANTE, R.; ASSIS, C.; MANSUR, J. M.; BARRETTO, A. C. P.; BELLOTTI, G.; PILEGGI, F. - Insuficiência da valva mitral por endomiocardiofibrose do ventrículo esquerdo: relato de caso. Arq. Bras. Cardiol., 52: 275-277, 1989

\section{Discussão}

\section{DR. CARLOS R. MORAES \\ Recife, PE}

O Dr. Sérgio Almeida de Oliveira e colaboradores merecem nossos elogios, por dois motivos principais. Primeiro, apresentam uma série consecutiva de 25 pacientes operados de endomiocardiofibrose (EMF) com mortalidade de $4 \%$. Isso contrasta com nossa experiência pessoal e com todos os trabalhos da literatura, inclusive do próprio InCor, ainda se relata mortalidade média de $18,8 \%$. Ora, admitindo-se que parte da operação proposta pelo $\mathrm{Dr}$. Sérgio é igual ao que todos os cirurgiōes têm realizado, referindo-me a endocardiectomia, temos que admitir que o segredo está na preservação da valva. Por outro lado, por mais perfeita que seja uma anuloplastia, o resultado hemodinâmico, no que diz respeito à competência valvar, não pode ser melhor ao funcionamento de uma bioprótese. Conclue-se, portanto, que seria a presença de prótese numa cavidade ventricular pequena a causa de todos os problemas. Em nossa experiência, observamos uma dramática redução da mortalidade quando passamos a utilizar próteses de baixo perfil e de diâmetros menores. No presente trabalho, os autores não especulam sobre a (ou as) causas que determinam uma baixa mortalidade na série apresentada, e eu gostaria de ouvir a opinião do Dr. Sérgio sobre $O$ assunto. $O$ segundo motivo pelo qual eu me congratulo com o Dr. Sérgio diz respeito ao fato de ter conseguido preservar todas as valvas numa série consecutiva de 25 casos. Desde que Nair e colaboradores, em 1980, demonstraram ser possível preservar a valva mitral na EMF, temos tido essa sorte de preocupação. Entretanto, em contraste com o Dr. Sérgio, que conseguiu preservar $100 \%$ das valvas, em nossa experiência e na de outros cirurgiōes isso tem sido possível em cerca de $12 \%$ dos casos. Como a EMF é uma doença misteriosa e que apresenta características regionais diferentes, a minha única dúvida é se realmente estamos tratando o mesmo tipo de doente. Por exemplo, em um caso operado recentemente e quando já conhecíamos o trabaIho do Dr. Sérgio, fomos incapazes de identificar estrutura valvar mitral que permitisse uma anuloplastia. Muito obrigado, e meus parabéns ao Dr. Sérgio e sua equipe.

\section{DR. JOSÉ WANDERLEY NETO Maceió, $A L$}

À partir de Dubost, em 1971, abriu-se uma nova perspectiva para o tratamento cirúrgico da endomiocardiofibrose com a decorticação ventricular e substituição da valva correspondente. Coube ao Prof. Carlos Moraes, do Recife, divulgar a técnica entre nós e, junto com seu grupo, dar muitas contribuiçōes para o estudo da doença. Esta técnica permite a recuperação funcional em muitos casos. Agora, entre nós, o Prof. Sérgio Oliveira, com esta inovação técnica, nos traz, sem dúvida, um grande avanço para o tratamento da doença. A técnica permite que possamos conservar as valvas atrioventriculares $e$ seus papilares, elementos importantes na manutenção de função ventricular a curto e longo prazo. Isto permite que indiquemos o tratamento cirúrgico nas formas iniciais da doença, quando esta se encontra restrita ao endocárdio e não há comprometimento de outros órgāos. Em Maceió, durante o período de dez anos, tivemos oportunidade de operar 16 casos de endomiocardiofibrose. Nos últimos três pacientes, utilizamos a técnica preconizada pelo Prof. Sérgio Oliveira: um caso de ventrículo esquerdo e outro de ventrículo direito; $O$ terceiro caso era biventricular e foi a óbito em baixo débito. A necropsia mostrou que havia uma intensa fibrose invadindo o miocárdio. Parabenizo o Prof. Sérgio, por mais esta contribuição à cirurgia cardíaca do nosso País. Muito obrigado à direção do Congresso e à atenção dos senhores.

\section{DR. OLIVEIRA \\ (Encerrando)}

Agradeço a todos os colegas que nos prestigiaram com seus comentários ao nosso trabalho. O Dr. Carlos Roberto de Moraes é, indiscutivelmente, a pessoa que mais contribuiu para a difusão dos conhecimentos sobre a cirurgia da EMF em nosso meio. Nós concordamos com sua dúvida sobre se a EMF é uma mesma doença, homogênea em sua expressão anatômica, assim como 
OliveIRA, S. A.; DALLAN, L. A.; PEREIRA-BARRETO, A. C.; MADY, C.; PILEGGI, F.; JATENE, A. D. - Endomiocardiofibrose: resultados do tratamento cirúrgico com conservação das valvas atrioventriculares. Rev. Bras. Cir. Cardiovasc., 6(1): 3-10, 1991.

em sua etiopatogenia. O que nós sabemos é que, em suas múltiplas formas de apresentaçāo, a fibrose endocárdica, felizmente, respeita, na grande maioria dos casos, as cúspides das valvas atrioventriculares, havendo apenas acometimento dos músculos papilares. A queda na mortalidade operatória, em nossa casuística, já se observava mesmo antes da introdução desta nova técnica, onde a mortalidade caiu de $20 \%$ para aproximadamente $10 \%$. Mas, com a cirurgia conservadora, houve melhora ainda mais expressiva, refletindo, provavelmente, melhor resultado funcional. O Dr. José Wanderley Neto referiu-se à sua experiência inicial com esta nova técnica e sugere a possibilidade de ser indicada a cirurgia em fases mais precoces da evoluçāo da doença, considerando-se a alta probabilidade de ser um procedimento conservador das valvas atrioventriculares. Nós temos cogitado desta mudança na indicação, mas os trabalhos clínicos realizados no InCor têm mostrado que os pacientes que estão em classe funcional I e II (NYHA) apresentaram boa evoluçăo clínica num seguimento de até 10 anos. Ao contrário, os pacientes em classe funcional III e IV apresentaram taxas de mortalidade. Por isto, ainda estamos reservando o tratamento cirúrgico para os pacientes com classe funcional III-IV. Agradeço, também, ao Dr. José Teles de Mendonça, pelos comentários e observaçōes sobre nosso trabalho. Muito obrigado. 